\title{
Psychiatric sequelae of acquired brain injury
}

\author{
Ken Barrett
}

Improvements over recent decades in acute care of the brain-injured now ensure that all but the most severely injured survive. The legacy of that survival is an increasing number of people with enduring organic mental disorders. Here, I will focus upon the psychiatric sequelae of five types of severe singleinsult brain injury: head trauma, subarachnoid haemorrhage, and hypoxic, hypoglycaemic and postencephalitic injury. Thrombotic stroke is a common and important cause of brain injury but the psychiatric consequences have been more extensively studied and are fairly well-known (Robinson \& Price, 1982; Starkstein et al, 1987). Damage due to alcohol and associated malnutrition is also well-documented (Jacobson \& Lishman, 1987, 1990). Neither will be specifically addressed here, though some of the consequences of brain injury are similar, regardless of cause.

\section{Five causes of brain injury}

\section{Head trauma}

About 1 in 10000 people each year (Medical Disability Society, 1988) suffers severe and persistent disability as a result of head trauma. Head trauma can lead to varying patterns of brain injury. Diffuse axonal injury is most likely to occur when there is rapid deceleration, leading to oscillating compression waves within the brain substance. This type of injury is common during road traffic accidents in which the head itself may appear relatively unmarked. Penetrating injury can occur due to a missile such as a gunshot, but in the UK occurs more commonly when the head strikes a hard object which breaches the skull and meninges and penetrates the brain. This type of injury carries the highest risk of posttraumatic epilepsy. Relatively small areas of brain damage in the brain stem, usually arising from extreme flexion or extension injuries of the neck, can result in very prolonged periods of coma followed by a range of enduring sensory, pyramidal and bulbar problems, but higher cortical functions may be intact. Head trauma sets in train a range of physiological processes which can be and often are more damaging than the immediate traumatic injury. This includes cerebral haemorrhage and oedema, with the risk of coning and respiratory arrest. Massive release of neurotransmitters, particularly glutamate, leads to an opening of calcium channels with cytotoxic consequences. There are now a variety of strategies for limiting the damage caused by these processes and this has lead to higher rates of survival, though with ever new patterns of severe and lasting damage. Repeated minor head trauma, as occurs in boxing, can also lead to brain damage, particularly in the basal ganglia (Corsellis, 1989). The contribution of brain damage to chronic postconcussive syndromes following a single minor head injury remains uncertain (King, 1997).

\section{Subarachnoid haemorrhage}

Subarachnoid heamorrhage (SAH) can occur as a consequence of head injury but also occurs spontaneously due to rupture of an arterial aneurysm, leakage of an arterio-venous malformation or other cerebral pathology. The annual incidence is about 6 per 100000 of the population (Storey, 1972). Aneurysms can occur in many locations but the most

Ken Barrett has been a consultant neuropsychiatrist for 10 years and is also Senior Clinical Lecturer in the Department of Psychiatry, Keele University. His main interest is in chronic neuropsychiatric disorders. The Neurobehavioural Unit in Stoke (Haywood Hospital, High Lane, Burslem, Stoke-on-Trent ST6 7AG) is one of few NHS units specialising in psychiatric problems following brain injury. He also chairs the UK Psychiatrists Brain Injury Group. 
common is at the junction of arteries in the circle of Willis which sits below the frontal lobes. Brain injury occurs following aneurysm in a number of ways: for example, a large volume of blood can cause local pressure, midline shift or coning; or arterial spasm can result in hypoperfusion for several hours resulting in hypoxic damage. The inferior and medial frontal lobes and medial temporal structures are most commonly damaged and for this reason psychiatric sequelae are relatively common, particularly personality change and depression. In addition, a wide range of physical disabilities can result, including visual deficits and epilepsy.

\section{Hypoglycaemic and hypoxic injury}

Hypoglycaemic brain damage occurs owing to accidental or deliberate insulin overdose. Hypoxia can have many causes including cardiac arrest, drowning and choking, but non-accidental injuries including hanging and carbon monoxide poisoning are also common. Hence, in many people who have hypoxic or hypoglycaemic injury the mental state immediately prior to injury is often one of depression with suicidal features. The annual incidence of these forms of injury is not known. The areas of brain that are most vulnerable to hypoxic/hypoglycaemic damage are those that have high metabolic rates. The hippocampi and basal ganglia are particularly sensitive to damage and, while attention and memory problems are the most common after-effects, many odd behavioural syndromes can develop including bizarre dissociative disorders (Eames, 1992). Cardiac arrest with subsequent resuscitation can lead to damage in areas of the brain with less rich circulation (the 'watershed areas') and the consequences include complex 'transcortical' dysphasias.

\section{Encephalitis}

Herpes simplex encephalitis has an annual incidence of about 1 per 1000000 of the population per year and is often identified and treated only when the illness is well-established. Illness suggestive of encephalitis in which no specific pathogen is found is probably more common. Herpes simplex can cause focal inflammation and damage in any area of the brain but the most common area to suffer the most severe damage is the medial temporal area. At its most severe this can lead to profound memory and concentration problems, and even 'Kluver-Bucy syndrome' including agnosia, hyperorality, agitation and altered sexual behaviour. This mix of symptoms and signs of varying severity is referred to as the 'postencephalitic syndrome' in ICD10 (World Health Organization, 1992).

\section{Psychiatric disorders following brain injury}

There are a number of reasons why brain injury may increase the risk of developing mental illness. Brain injury may: increase the constitutional vulnerability to major mental illnesses; impair the ability to adjust and adapt to life change; lead to a loss of role and employment, break-up relationships and produce financial hardship. There may also be persistent physical disabilities such as hemiparesis, ataxia, visual field or hearing loss, aphasia and epilepsy. Because of the wide range of complex problems that follow brain injury the Care Programme Approach (CPA) provides a very useful management framework. Box 1 summarises the main questions to be considered in assessing people with brain injury. Box 2 presents the classification of the psychiatric sequelae of brain injury according to ICD-10. This paper will concentrate upon organic affective disorder, organic delusional and schizophrenia-like disorders and organic personality disorder. First, coma and delirium will be discussed.

\section{Coma and delirium}

A 35-year-old man suffered a head injury in a road traffic accident and was in coma for three days. On recovering consciousness he became restless and aggressive. He did not believe he was in hospital, said the nurses were trying to kill him and attempted to leave. He was assessed by a psychiatrist who found him to be disoriented in time and place and acutely anxious about the harm he believed was about to be done to him. He knew that he had suffered a head injury but believed he was deliberately run down. He was detained in hospital under a section of the Mental Health Act 1983 and was transferred to an acute psychiatric ward. He appeared less anxious in that environment and received only two doses of a major tranquillizer. He settled quickly thereafter and after three days, had become fully oriented. There were no psychotic features hence he was discharged from his section.

Coma is caused by dysfunction of the reticular activating system (RAS) in the brain stem. This can occur as a result of local compression or for metabolic reasons. The period of coma ends when RAS function resumes. In the transition to full alertness there will be a period of drowsiness and inattention (i.e. delirium). During this period of transition some people are quiet and docile but many are not and a wide range of behavioural, emotional, perceptual abnormalities can occur. These include elation, emotionality, irritability and aggression, feelings of persecution, perceptual misinterpretations or hallucinations. The 
mental state during this period of post-traumatic delirium can change every few hours and the state may resolve quickly. The precise cause of the delirium is sometimes unclear and it is easy to miss a case of alcohol or tranquillizer withdrawal in the aftermath of head or multiple injury. Electrolyte disturbance, particularly hyponatraemia, may contribute, although this is more common after neurosurgery. A pre-existing psychotic illness may also confuse the clinical picture and post-injury delirium sometimes evolves into a manic or paranoid state.

Behaviour disorders in the phase following coma present a major problem for neurosurgical staff and

Box 1. Assessing people with psychiatric problems following acquired brain injury

Lookfor evidence of:

Organic delusional, affective disorder or adjustment disorder

Organic personality disorder or less severe personality change (in particular, abulia and impulsive behaviours including aggression, bizarre or eccentric behaviours)

Significant risk of deliberate or accidental self-harm or harm to others

Significant motor (weakness, spasticity, ataxia) or sensory/perceptual (visual, auditory, somatosensory, taste and smell, neglect or inattention) deficit

Impairment in verbal communication (expressive and receptive dysphasias, dyslexia particularly associated with left-sided brain damage)

Impairment in non-verbal communication (inability to use or interpret emotional tone of voice and facial expression; inability to understand humour, irony, sarcasm particularly associated with right-sided brain dysfunction)

Cognitive dysfunction (attention and concentration, visual, verbal, procedural and episodic memory, sequencing, planning, problem-solving/cognitive slowing or inability to carry out several tasks simultaneously)

Problems pre-injury (in mental health, learning, personality traits, social skills, relationships and employment)

Significant relationship problems (spouse, parents, children)

Social welfare needs (housing, finance, transport, home aids and adaptations, home care, day care or respite care) psychiatric assistance is frequently sought. The psychiatrist's role is to establish whether or not diagnoses other than delirium are present (e.g. a preexisting psychosis or major depressive disorder) and whether factors other than the brain injury may be contributing (e.g. metabolic disturbance, alcohol or tranquillizer withdrawal), and to advise on or take over the management of emotional sequelae. Restlessness is the most common problematic symptom in post-injury delirium and, although it may settle within hours, can cause major problems in a surgical or medical environment. Relatives are understandably apprehensive about having the person home owing to the need for 24-hour observation. Removal from that environment to a more domestic one with trained mental health care staff can have beneficial effects and avoid the need to prescribe neuroleptics which may impede recovery (Feeney et al, 1982). These problems are undoubtedly acute and psychiatric in nature, yet many acute psychiatric services are reluctant to take such patients because their problem is 'organic' or because they are considered 'at risk' in an acute ward. Neither reason is valid as in the absence of a local specialist neuropsychiatry service such problems are within general adult psychiatry. That said, it is also common for relatives to be resistant to transfer to psychiatric care.

Box 2. ICD-10 classification: sequelae of acquired brain injury

Organic hallucinosis

Organic catatonic disorder

Organic delusional (including schizophrenia-like) disorder

Organic mood (affective) disorder

Organic dissociative disorder

Organic labile disorder

Organic personality disorder

Mild cognitive disorder

Post-encephalitic syndrome

Post-concussional syndrome

Other organic personality and behaviour disorders due to brain damage

Delirium, not induced by alcohol and other psychoactive substances

Dementia in other specified diseases classified elsewhere

Unspecified dementia

Organic amnesic syndrome, not induced by alcohol and other psychoactive substances

Other specified mental disorders due to brain damage 
Delirium often only lasts a few hours or days and reduction of agitation and related behaviours may be achieved with sedative medication alone. Chlormethiazole and clonazepam can be useful for up to 3-4 days in this context. Porcher et al (1994) reported that buspirone in combination with carbamazepine was effective in reducing agitation in four patients in post-injury delirium. Carbamazepine alone may also be effective. Major tranquillizers must be seen as a last resort and used for as a short period of time as possible. Butyrophenones rather than phenothiazines are indicated.

\section{Organic affective disorder}

\section{Depression}

A 39-year-old teacher suffered a severe head injury in a road traffic accident. He was in coma and ventilated for seven days and suffered a severe and nearly fatal chest infection. He recovered consciousness after 10 days and emerged from delirium at three weeks. He had no focal motor or sensory deficits but had a range of cognitive impairments which gradually improved in the months after injury. Previously confident and flamboyant, he had become timid and reserved.

He returned to teaching after a year, although with a reduced load and a greatly increased dependence upon notes and cues. He coped less well with staff interactions and meetings and became more socially withdrawn. By half-term he was persistently low in mood, irritable, gloomy about the future and sleeping 12 hours out of 24 at weekends. He was referred for psychiatric opinion by his general practitioner and was initially treated with supportive counselling, without benefit. His mood significantly improved two weeks after commencing antidepressant medication but he remained off work for several months and eventually decided to retire from teaching.

The frequency of depressive disorder after head trauma has been reported to be as high as $50 \%$ (Gualtieri \& Cox, 1991), and up to $25 \%$ following SAH (Storey, 1972) but the findings of different investigators vary widely depending upon sample size, diagnostic criteria, severity of, and time since, injury. No figures are available for the prevalence of depression in post-encephalitic, hypoxic and hypoglycaemic damage. Jorge et al (1993), employing standardised assessments and DSM-III-R criteria, found $23 \%$ of a sample of severely head-injured patients met criteria for major depression at three months post-injury, and the rate fell only slightly through the first year. However, in the first three months after trauma, sleep, appetite, vitality, temper and other areas of function are commonly impaired as a direct result of neuronal damage and so the application of standardised psychiatric criteria may be misleading. None the less, depression is an important contributor to disability after injury and should be considered, particularly when there is a deterioration in function in an injured patient who had been improving. In the longer term a number of factors can make it difficult for professionals and family members to recognise the presence of depressive disorder. The expression of emotion in facial expression and tone of voice may be impaired by right hemisphere, basal ganglia and thalamic damage. Dysphasia and dysarthria can make it difficult to understand what is reported, and affective features of depressive disorder can be misidentified as nothing more than understandable unhappiness in the face of hardship. Persistent low mood, anhedonia, irritability and poor motivation, with or without other features of depressive disorder, should lead to the consideration of a trial of treatment with an antidepressant, particularly when the mental state or social function has deteriorated.

A pre-injury history of depressive disorder and poor social function appear to be the most important risk factors for post-injury depression (Robinson \& Jorge, 1994) and brain injury not infrequently arises as a result of self-harm in the context of depression. The location of focal brain lesions may also be a factor in the development of depression but separate studies have implicated lesions in the right frontal (Grafman et al, 1986) and left frontal (Jorge et al, 1993) areas. In practice, most civilian brain injury is diffuse and the neurotransmitter changes that underlie clinically significant depressive disorders are likely to be modulated by many factors.

Response to antidepressants is often satisfactory and the choice of medication should be guided by the desire to avoid side-effects. Brain injury increases sensitivity to sedative effects, anticholinergic effects may impair concentration, and there is an increased risk of epilepsy. Hence it is best to avoid tricyclics in the first instance and a selective serotonin reuptake inhibitor is the treatment of choice. It is worth noting that low-dose sedative tricyclics, particularly amitriptyline $(10-25 \mathrm{mg}$ ) can be valuable in post-injury headache and sleep-wake cycle problems (i.e. where the cycle of sleep is delayed or irregular). Treatmentresistant depression should be treated as in noninjured people. Although electroconvulsive therapy (ECT) is not contraindicated there are few data on effects of ECT in people with severe brain injury and it is best avoided in the first six months after injury.

In the aftermath of a severe brain injury many aspects of the injured person's life are affected. The ability to engage in employment and leisure pursuits may be impaired and personality change can lead to the breakdown of relationships and social networks, with increased dependence upon parents 
or spouse. Levels of carer distress, anxiety and depression are high even five years after injury (Brooks, 1990). Psychological support for the injured person and their family, and practical help with finances and other needs are also important components of treatment. Cognitive-behavioural and other psychological therapies have been reported to be effective in individual cases (Miller, 1991; Prigatano, 1991).

\section{Mania}

A 48-year-old engineer fell while rock climbing and suffered a blow to his right temple. He was only briefly unconscious but subsequently developed a subdural haematoma which required surgical drainage. A computed tomography scan revealed right temporo-parietal damage. He recovered consciousness rapidly after surgery and was oriented within three days but was restless, had pressure of talk and flight of ideas, was over-familiar, elated and at times irritable, and had impaired sleep. He had no previous history of psychiatric disorder. He settled quickly on carbamazepine $400 \mathrm{mg} /$ day and the medication was reduced and withdrawn a month after injury without recurrence of mania.

Brain injury in childhood has been reported in 5\% of people with adult-onset mania (Wilcox \& Nasrallah, 1987). However, the term 'organic mania' is usually applied to manic states which follow brain injury in adulthood. There are over 30 cases of mania following brain injury in the literature, including manic states that merge with resolving posttraumatic delirium (e.g. Bracken, 1987; Starkstein, 1988). Organic mania usually develops in the presence of right hemisphere lesions, particularly when the basal temporal region is injured. The diagnosis is complicated in the early post-injury period by the fact that restlessness and inattention are common in delirium, and in later stages by disinhibition and fatuousness due to frontal injury. Organic mania can be more confidently diagnosed when the injured person has regained orientation but their mental state has altered to include elation, irritability, flight of ideas and pressure of talk. Several treatment approaches are reported in the literature, all on a single case, open trial basis. Carbamazepine is probably the treatment of first choice but a more rapid titration may be necessary than in the treatment of epilepsy (200-400 mg start dose). Valproate, lithium and major tranquillizers have also been reported to be effective. There is no literature on the longer-term prognosis in organic mania, but in the first instance it is appropriate to assume the episode is a one-off, and gradually withdraw medication. When mania immediately follows delirium a gradual reduction can be attempted after only a few weeks.

\section{Organic delusional and schizophrenia-like disorders}

A 23-year-old accountant suffered a severe head injury in a road traffic accident. He was in coma for three weeks and in post-traumatic delirium for three months. Computed tomography brain scan showed extensive inferior bi-frontal damage. Thereafter, he made a remarkable physical recovery although had a moderate a global reduction in intellect, a mild dysarthria and flattened voice intonation, personality change and limited insight into his disabilities. He expressed doubt about ever suffering a head injury and began to accuse family members of cheating him in various ways.

He was referred to a clinical psychologist by a medical rehabilitationist who sought to help him accept and adjust to his disabilities. However, he maintained that he would return to his old job and became increasingly irritable, talking of little else but the wrongs he believed had been done to him, particularly by his brother. He eventually threatened his brother with a knife, was arrested and subsequently detained under the Mental Health Act 1983.

He was considered to be suffering from paranoid delusions, and he improved greatly over a period of six weeks on sulpiride but was over-sedated and so this was replaced with risperidone. He later admitted that he had been hearing voices telling him that he had been 'ripped off' for at least two years and that he often heard people in the street or pubs talking about him. These experiences had stopped after three weeks on the medication and he then recognised that they were abnormal, although his insight into his limitations with regard to employment did not improve. He remained stable after two years on $3 \mathrm{mg}$ risperidone daily, although attempts to reduce the dosage have been followed by a return of paranoid ideation.

Several studies have shown high rates of psychosis in people who have suffered brain injury. Davison \& Bagley (1969) after an extensive literature review concluded that brain injury increased the risk twoto three-fold, and subsequent research has not altered this figure convincingly. While there is also evidence that left temporal leions are more likely to be associated with schizophreniform disorders (Buckley et al, 1993), most causes of brain injury do not restrict damage to a single lobe. A number of factors can complicate the assessment and diagnosis of psychosis in brain-injured people. In psychosis there is an abnormality in individuals' ability to correctly interpret what is happening within them and in the world around them. Brain injury can alter their appraisal of the world in a variety of ways that are listed in Box 3, and this should be borne in mind when assessing such patients for possible psychosis. The identification of delusional disorders may also be impaired by acceptance of aberrant behaviour as due to injury-induced eccentricity of personality. 
There is almost no literature addressing the efficacy of antipsychotic treatment in organic delusional disorder, and brain-injured people are often rejected from clinical trials of new drugs. While there have been several single and multiple case reports of post-injury psychosis in the past 30 years only three included detail of response to treatment. Two cases responded well to neuroleptics (Nasrallah et al, 1981; Vedie et al, 1993 ) and one did not but responded to carbamazepine (Ritchie et al, 1989). Based upon his extensive clinical experience Lishman (1998) maintains that most post-injury psychoses respond to antipsychotic drugs and this is certainly my experience, but optimum management should include a care programme that addresses the wide range of problems that can arise as a result of brain-injury and psychosis. In addition, people with brain injury are at increased risk of drug side-effects including early and late extrapyramidal effects, sedation, reduced motivation, and epileptic seizures. When hemiparesis has occurred, dopamineblocking drugs can produce or exacerbate spacticity as well as impairing gait and coordination. Anticholinergic drugs used to treat side-effects are also more likely to impair concentration and memory in brain-injured people. Sulpiride or risperidone are in my experience usually effective but these are not entirely free from extrapyramidal effects and there may be a case for considering newer 'atypical' agents such as olanzapine and quetiapine as a first line.

A 43-year-old male lorry driver suffered a compound depressed fracture of the right temporo-parietal region. Several bony fragments were removed from the underlying brain and he was left with a large skull defect. He recovered consciousness after three days and was immediately very restless and assaultive, and this state only settled with major tranquillizers. He became oriented after six weeks and insisted on being discharged from hospital. Several attempts to withdraw neuroleptics led to anxiety, agitation and irritability, but they were eventually withdrawn following the development of an oral dyskinesia two years after his injury.

He subsequently became depressed, anxious and avoidant, and was inclined to stay in bed when at home. Respite and review admissions occurred and during these he appeared quickly to brighten up, participated in ward activities and denied feeling depressed. His behaviour at home remained the same and after some time he talked of suicide and appeared threatening to his wife and adult step-sons. He agreed to admission and on this occasion confessed that he believed his wife and step-sons were planning to kill him, and had felt this since his injury. He responded rapidly to olanzapine $20 \mathrm{mg}$ daily, was discharged after three weeks and did not revert to his previous behaviour.

Firm recommendations regarding these newer agents cannot be given until studies of their positive and adverse effects have been conducted in braininjured people. When psychosis fails to respond to antipsychotics a trial of carbamazepine or valproate may be worthwhile. No information is available on the frequency of treatment resistance or the longterm prognosis in post-injury psychosis.

\section{Organic personality disorder}

One of the most common complaints voiced by relatives of brain injured people is that their loved one's

Box 3. Factors contributing to impaired appraisal of the world after brain injury

Memory impairment may include not only the ability to learn new information, but also the ability to recall old information. This can include autobiographical details and several years in an individual's life may be forgotten. Hence, for example, retirement from work or remarriage may have been forgotten, with predictable results. Problems with autobiograpical memory are particularly common after right dorso-lateral frontal lobe injury

Perceptual and sensory problems can impair the ability to see, hear etc., but there may also be sensory neglect, inattention or anosagnosia-denial of illness

Impaired self-awareness and understanding of other's reactions can occur, particularly following basal frontal lobe lesions

In receptive aphasia inability to understand what is being said is often put down to a fault in the person speaking. The affected individual may also be unaware that they themselves are talking nonsense

In receptive aprosodia the ability to understand changes in facial expression and voice intonation (non-verbal communication) is impaired or lost so what is said is taken literally. Hence loss of understanding of humour, sarcasm, irony etc. Right parietotemporal lesions can cause these problems

In agnosia objects and their purpose cannot be correctly identified and this can lead to a wide range apparently odd behaviours

Complex partial seizures can produce a wide range of short-lived but disturbing sensory and emotional experiences including stereotyped hallucinations and complex dream-like experiences 
personality has changed. Sometimes this is an exaggeration of previous personality traits but often the injured individual is described as being like a different person. Occasionally this change is seen as an improvement, but I have only seen this when a previously anxious, worry-prone and obsessional individual becomes more relaxed and laid back, usually as a result of predominantly focal left frontal lobe damage. Although any constellation of personality traits can arise after brain injury, three are most commonly complained of: disinhibited behaviour, explosive temper problems and abulia (impaired drive and motivation - literally an absence of will).

\section{Temper disorders and disinhibition}

In recent times Blumer \& Benson (1975) were the first to point out that subtypes could be identified within the frontal lobe syndrome. Orbital frontal and anterior temporal damage results in a range of disinhibited behaviour of various types, often associated with a reduction in emotional warmth and lack of insight. In some cases disinhibition is confined to a temper disorder. Although temper disorders can occur primarily due to damage to these brain structures, they are, in most cases, multifactorial in origin and can be complex to manage. In managing these disorders it is useful to identify if there is a warning, trigger or precipitant. Is there recollection of the outburst, and remorse afterwards? Many factors can contribute to the development and maintainance of temper problems including: depressive disorder; misinterpretation of others' behaviour based upon aprosodia, psychosis or impaired cognition; psychosocial stressors including marital/family disharmony and financial pressures; and physical factors including seizure disorders, pain, alcohol and other drug use (minor tranquillizers such as diazepam may increase dyscontrol). Poorly controlled epilepsy may also be associated with temper problems, presumably due to neurophysiological dysfunction in temporal and frontal lobe structures that are important for behavioural inhibition.

A number of drug treatments have been used to reduce aggression following brain injury. There have been case reports of carbamazepine in temper disorders following brain injury (e.g. Lewin \& Sumners, 1992) and a single case report using valproate (Geracioti, 1994). It is not known if the anticonvulsant or psychotropic properties of these drugs lead to their beneficial effects. Occasionally, where carbamazepine produces sedative effects, it can impair temper control. Minor tranquillizers may also worsen dyscontrol.

Propranolol can be useful and may also reduce both agitation and irritability at a wide dosage range from $40 \mathrm{mg}$ daily upwards (Greendyke et al, 1986; Brooke et al, 1992). Major tranquillizers should be a last rather than first resort in the treatment of temper disorders unless psychosis is present or suspected.

Disinhibited behaviours of various sorts may be amenable to behavioural strategies but consistent management - and in severe cases, a specialist residential setting - will be necessary. Some centres report success with a combination of behavioural strategies in association with drug treatment (Eames \& Wood, 1981) although generalisation to nonresidential settings is difficult to achieve. A useful review is provided by Corrigen \& Jakus (1994).

\section{Abulia}

Abulia occurs when there is damage to the medial frontal lobe structures. This is believed to occur because of damage to a dopaminergic system with its origin in the midbrain. Hence, damage to the midbrain and the lateral hypothalamus can have the same result (Fisher, 1983). At its most severe the result of such damage is an organic catatonic state, but less severe abulic states are common and often overlooked. The first description of drug treatment for abulia was provided by OliverSacks (1973) in his book Awakenings. L-dopa produced transient but dramatic improvements in drive in people with abulia as a result of encephalitis lethargica. Ross \& Stewart (1981) provided the first description of the use of a dopamine agonist, bromocriptine, in the treatment of severe abulia, and I (Barrett, 1991) described four cases of less severe abulia which were responsive to bromocriptine or lysuride. There have since been a number of other reports using this and other dopamine agonists, and although not licensed for this indication bromocriptine is now widely used in brain injury rehabilitation. There are a number of potential problems with the use of dopamine agonists (see Box 4), and these should be borne in mind. That said, dopamine agonists can, when used cautiously and appropriately, dramatically reduce disability in people with no other hope of improvement.

\section{References}

Barrett, K. (1991) Treating organic abulia with bromcriptine and lisuride. Journal of Neurological and Neurosurgical Psychiatry, 54, 718-721.

Blumer, D. \& Benson, F. (1975) Personality change with frontal and temporal lobe lesions. In Psychiatric Aspects of Neurologic Disease (eds D. F. Benson \& D. Blumer), pp. 151-171. New York: Grune \& Stratton.

Bracken, P. (1987) Mania following head injury. British Journal of Psychiatry, 150, 690-692.

British Medical Association \& Royal Pharmaceutical Society of Great Britain (1999) British National Formulary. London: BMA and The Pharmaceutical Press.

Brooke, M. M., Patterson, D. R., Questad, K, A., et al (1992) The treatment of agitation during initial hospitalisation after traumatic brain injury. Archives of Physical Medicine and Rehabilitation, 73, 917-921. 
Brooks, N. (1990) The head injured family. Journal of Clinical and Experimental Neuropsychology, 13, 1-34.

Buckley, P., Stack, J. P., Madigan, C., et al (1993) Magnetic resonance imaging of schizophrenia-like psychoses associated with cerebral trauma: clinico pathological correlates. American Journal of Psychiatry, 150, 146-148.

Corrigan, P. W. \& Jakus, M. R. (1994) Behavioural treatment. In Neuropsychiatry of Traumatic Brain Injury (eds J. M. Silver S. C. Yudofsky \& R. E. Hales), pp. 733-769. Washington, DC: American Psychiatric Press.

Corsellis, J. (1989) Boxing and the brain. British Medical Journal, 134, 105-109.

Davison, K. \& Bagley, C. R. (1969) Schizophrenia-like psychoses associated with organic disorders of the central nervous system: a review of the literature. In Current Problems in Neuropsychiatry: Schizophrenia, Epilepsy, the Temporal Lobe (ed. R. N. Herrington), pp. 113-184. British Journal of Psychiatry special publication, no. 4. Ashford: Headley Brothers.

Eames, P. (1992) Hysteria following brain injury. Journal of Neurological and Neurosurgical Psychiatry, 55, 1046-1053.

- \& Wood, R. (1981) Rehabilitation after severe brain injury: a follow-up study of a behaviour modification approach. Journal of Neurological and Neurosurgical Psychiatry, 48, 613-619.

Feeney, Gonzales, A., Law, W. A., et al (1982) Amphetamine, haloperidol and experience interact to affect rate of recovery after motor cortex injury. Science, 217, 855-857.

Fisher, C. M. (1983) Abulia monor vs agitated behaviour Clinical Neurosurgery, 31, 9-31.

Geracioti, T. D. (1994) Valproic acid treatment of episodic explosiveness related to brain injury. Journal of Clinical Psychiatry, 55, 416-417.

Grafman, J., Vance, S. C., Swingartner, H., et al (1986) The effects of lateralised frontal lesions on mood regulation. Brain, 109, 1127-1148.

Greendyke, R. M., Shuster, D. B., Wooton, J. A., et al (1986) Propranolol in the treatment of assaultive patients with organic brain disease. A double blind study. Journal of Clinical Psychiatry, 8, 23-26.

Gualtieri, C. T. \& Cox, D. R. (1991) The delayed neurobehavioural sequalae of traumatic brain injury. Brain Injury, 5, 219-231.

Jacobson, R. R. \& Lishman, W. A. (1987) Selective memory loss and global intellectual deficits in alcoholic Korsakoff's syndrome. Psychological Medicine, 17, 649-655.
Jacobson, R. R. \& Lishman, W. A. (1987) Cortical and diencephalic legions in Korsakoff's syndrome: a clinical and CT scan study. Psychological Medicine, 20, 63-75.

Jorge, R., Robinson, R. G., Arndt, S. V., et al (1993) Depression and traumatic brain injury: a longitudinal study. Affective Disorders, 27, 233-243.

King, N. (1997) Mild head injury: neuropathology, sequelae, measurement and recovery. British Journal of Clinical Psychology, 36, 161-184.

Lewin, J. \& Sumners, D. (1992) Successful treatment of episodic dyscontrol with carbamazepine. British Journal of Psychiatry, 161, 261-262.

Lishman, W. A. (1998) Organic Psychiatry (3rd edn). London: Blackwell Science.

Medical Disability Society (1988) The Report of the Working Party on the Management of Traumatic Brain Injury. London: Medical Disability Society.

Miller, I. (1991) Psychotherapy of the brain-injured patient: principles and practices. Cognitive Rehabilitation, 9, 24-30.

Nasrallah, H. A., Fowler, R. C. \& Judd I. I. (1981) Schizophrenialike illness following head injury. Psychosomatics, 22, 359-396.

Porcher, E., Filteau, M. J., Bouchard, R. H., et al (1994) Efficacy of the combination of buspirone and carbamazepine in early posttraumatic delirium. American Journal of Psychiatry, 151, 150-151.

Prigatano, G. P. (1991) Disordered mind, wounded soul: the emerging role of psychotherapy in rehabilitation after brain injury. Journal of Head Trauma Rehabilitation, 64, 1-10.

Ritchie, E. C., Primelo, R., Radke, A. Q. (1989) Bullet in the brain: a case of organic psychosis. Journal of Neuropsychiatry and Clinical Neurosciences, 1, 449-451.

Robinson, R. G. \& Price, T. R. (1982) Post stroke depressive disorders: a follow-up study of 103 patients. Stroke, 13, 635-641.

Robinson, R. G. \& Jorge, R. (1994) Mood disorders. In Neuropsychiatry of Traumatic Brain Injury (eds J. M. Silver, S. C. Yudofsky \& R. E. Hale), pp. 219-250. Washington, DC: American Psychiatric Press.

Ross, E. D. \& Stewart, R. M. (1981) Akinetic mutism from hypothalamic damage: successful treatment with a dopamine agonist. Neurology, 31, 1435-1439.

Sacks, O. (1973) Awakenings. London: Duckworth.

Starkstein, S. E., Robinson, R. G. \& Price, T. R. (1987) Comparison of cortical and subcortical lesions in the production of post-stroke mood disorders. Brain, 110, 1045-1059.

\section{Box 4. Potential problems with use of dopamine agonists in brain injury rehabilitation}

Apathy and lack of spontaneity following brain injury most commonly occur within the context of a depressive disorder. Hence other symptoms of a depressive disorder should be sought in the first instance and antidepressant medication considered

Dopamine agonists can trigger a range of psychotic phenomena and should be used only with careful monitoring, particularly where there is a previous history of psychosis

Although the drugs are generally well-tolerated hypotension can occur and so a low dosage (e.g. $2.5 \mathrm{mg}$ bromocriptine nocte) should be used initially with a gradual increase up to British National Formulary (British Medical Association \& Royal Pharmaceutical Society of Great Britain, 1999) dose limits

An increase in spontaneous behaviours is sought, but resulting behaviours may be undesirable. I have had to stop medication on three occasions due to the development of increased sexual arousal and disinhibited sexual behaviour. In all cases there were also beneficial effects on other aspects of behaviour. In fact, where any problematic behaviour occurs in a person with abulia, dopamine agonists can make this behaviour worse

Abulia may be accompanied by severe cognitive impairment. An increase in drive and spontaneity can reduce the level of prompting and care required but may produce an increase in drive without the cognitive ability to direct it (hence a greater tendency to restlessness and wandering off)

Abulia may sometimes accompany significant physical disability and improving drive can have its dangers in this setting 
Starkstein, S. E., Boston, J. D. \& Robinson, R. G. (1988) Mechanisms of mania after brain injury. 12 cases and a review of the literature. Journal of Nervous and Mental Diseases, 176, 87-100.

Storey, P. B. (1972) Emotional disturbances before and after subarachnoid haemorrhage. In Physiological, Emotional and Psychosomatic Illness (Ciba Foundation Symposium No. 8) (eds R. Porter \& J. Knight), pp. 337-343. Amsterdam: Associated Scientific Publishers.

Wilcox, J. A. \& Nasrallah, H. A. (1987) Childhood head trauma and psychosis. Psychiatric Research, 21, 303-306.

World Health Organization (1992) The Tenth Revision of the International Classification of Diseases and Related Health Problems (ICD-10). Geneva: WHO.

Vedie, C., Battoum, H. \& Katz, G. (1993) Schizophrenie post-traumatique. La Presse Medicale, 22, 1091-1094.

\section{Multiple choice questions}

1. Depressive disorder following brain injury:

a rarely responds to antidepressant medication

b can be easily identified by carers and professionals

c may be the cause of a global deterioration in someone who was previously improving

d may be obscured by extrapyramidal problems and aprosodia.

2. Abulia following brain injury:

a is a form of movement disorder

b may occur in the context of a depressive disorder

c is generally improved by major tranquillizers

d may be improved by dopamine agonists such as bromocriptine.
3. Temper disorders following brain injury:

a are generally improved by minor tranquillizers

$b$ are often caused by factors other than malfunctioning brain

c should in the first instance be treated by major tranquillizers

$\mathrm{d}$ may arise in as a result of psychosis or depression.

4. Hypoglycaemic and hypoxic brain damage:

a often occur in someone who was depressed at the time of injury

b generally lead to a uniform pattern of brain damage

c often result in persistent memory problems

d can result in bizarre dissociative disorders.
MCQ answers

$\begin{array}{llllllll}\text { 1 } & & & \text { 2 } & & 3 & & 4 \\ \text { a } & \text { F } & \text { a } & \text { F } & \text { a } & \text { F } & \text { a } & \text { T } \\ \text { b } & \text { F } & \text { b } & \text { T } & \text { b } & \text { T } & \text { b } & \text { F } \\ \text { c } & \text { T } & \text { c } & \text { F } & \text { c } & \text { F } & \text { c } & \text { T } \\ \text { d } & \text { T } & \text { d } & \text { T } & \text { d } & \text { T } & \text { d } & \text { T }\end{array}$

\section{Commentary}

\section{Peter Eames}

\section{Causes of acquired brain injury}

It is helpful to consider two different kinds of acquired brain injury. The more common is stroke, which, like low-velocity penetrating injury, produces severe, often total destruction of a circumscribed area of brain substance. It is remarkable how rarely psychiatric help is sought for those with this kind of injury. The other kind is found in the injuries discussed by Ken Barrett: non-penetrating trauma, subarachnoid haemorrhage, hypoxia, hypoglycaemia and encephalitis. What distinguishes these is the combination of localised and diffuse insults,

Peter Eames is a consultant neuropsychiatrist at Grafton Manor Brain Injury Rehabilitation Unit (Grafton Regis, Towcester Northants, NN12 7SS). For 20 years, his main interests have been in acquired brain injury, in pursuit of which he helped develop the Kemsley Unit at St Andrew's Hospital and Grafton Manor, both units providing treatment and rehabilitation for those with severe behaviour disorders after brain injury. He has also worked in head injury follow-up clinics in district general hospitals. 